\title{
Observing Degradation of Visual Representations over Short Intervals When Medial Temporal Lobe Is Damaged
}

\author{
David E. Warren ${ }^{1}$, Melissa C. Duff ${ }^{1,2}$, Daniel Tranel ${ }^{1}$, \\ and Neal J. Cohen ${ }^{3}$
}

\begin{abstract}
Medial temporal lobe (MTL) contributions to the brief maintenance of visual representations were evaluated by studying a group of patients with MTL damage. Eye movements of patients and healthy comparison subjects were tracked while performing a visual search for a target among complex stimuli of varying similarity to that target. Despite the task having no imposed delays, patients were impaired behaviorally, and eye movement measures showed abnormally rapid degradation of target representations in the patients. Eye movement data
\end{abstract}

\section{INTRODUCTION}

Medial temporal lobe (MTL) structures play an acknowledged role in the formation and retrieval of new enduring declarative or relational memories (Eichenbaum \& Cohen, 2001; Cohen \& Squire, 1980; Scoville \& Milner, 1957), but recent findings from neuropsychological and neuroimaging studies indicate that MTL components may also contribute to representations that need only to be maintained or manipulated across a brief interval, a function conventionally associated with prefrontal, parietal, and inferior temporal cortices (cf. Jonides et al., 2008; Postle, 2006; Wager \& Smith, 2003). Deficits in patients with MTL damage have been observed at a variety of delays ranging from minutes (Ryan, Althoff, Whitlow, \& Cohen, 2000) to seconds (Ezzyat \& Olson, 2008; Shrager, Levy, Hopkins, \& Squire, 2008; Hannula, Ryan, Tranel, \& Cohen, 2007; Hannula, Tranel, \& Cohen, 2006; Nichols, Kao, Verfaellie, \& Gabrieli, 2006; Olson, Moore, Stark, \& Chatterjee, 2006; Olson, Page, Moore, Chatterjee, \& Verfaellie, 2006; Holdstock et al., 2002; Buffalo, Reber, \& Squire, 1998), for a range of stimulus materials including scenes (Hannula et al., 2006, 2007; Hartley et al., 2007; Lee, Buckley, et al., 2005; Lee, Bussey, et al., 2005; Ryan \& Cohen, 2004b; Ryan et al., 2000), faces (Shrager et al., 2008; Nichols et al., 2006; Lee, Buckley, et al., 2005;

\footnotetext{
${ }^{1}$ University of Iowa College of Medicine, ${ }^{2}$ University of Iowa,

${ }^{3}$ University of Illinois at Urbana-Champaign
}

showed a modulation of the duration of fixations as a function of the similarity of fixated array lures to the target, but the effect was attenuated in patients during long fixation paths away from the sample target. This effect manifested despite patients' shorter searches and more frequent fixations of the sample target. Novel techniques provided unique insight into visual representation without healthy MTL, which may support maintenance of information through hippocampal-dependent relational binding.

Lee, Bussey, et al., 2005), and other objects (Barense, Gaffan, \& Graham, 2007; Olson, Moore, et al., 2006; Lee, Buckley, et al., 2005; Lee, Bussey, et al., 2005; Buffalo et al., 1998). Likewise, neuroimaging studies show activation not only of prefrontal regions but also of the MTL during maintenance across short intervals of faces (Olsen et al., 2009; Ranganath \& D'Esposito, 2001), novel objects (Ranganath, Cohen, \& Brozinsky, 2005), and scenes (Hannula \& Ranganath, 2008). These findings converge to imply that the MTL supports the ongoing representation of new information, but the nature and time course of that information in the absence of MTL processing remains unspecified.

Taking the visuospatial domain as an example, it has been shown that MTL damage impairs memory performance for photographic or computer-rendered scenes even at the shortest of delays. Focal MTL lesions changed the manner in which patients looked at relationally manipulated versions of previously studied scenes (involving rearrangement of scene elements) at both long (Ryan et al., 2000) and short delays (Ryan \& Cohen, 2004a, 2004b), disrupted the ability to represent spatial and associative relations among stimuli even when the time between study and test was best measured in seconds (Hannula et al., 2006, 2007), and impaired simultaneous comparison of scenes viewed from different perspectives (Hartley et al., 2007; Lee, Buckley, et al., 2005; Lee, Bussey, et al., 2005). Deficits after short delays have been observed not only with the rich visual scenes in the above-cited experiments but also with arrays of simple shapes (Shrager et al., 2008; Olson, Moore, et al., 2006; Holdstock et al., 2002). 
These results indicate that the MTL plays a crucial role not just for enduring memories but also for representations that need to persist only long enough to satisfy the demands of ongoing processing employing on-line representations (i.e., what have been called on-line representations; see Barense et al., 2007; Ryan \& Cohen, 2004a). Scenes or other relationally complex displays are of particular interest in this context because, unless all that is needed is a rapid, holistic appreciation of a scene's gist (e.g., identifying a briefly presented image as a beach or an office; see Intraub, 1980), processing of scene-like materials entails the serial identification of the various constituent scene elements and their spatial arrangement (i.e., piecemeal construction and maintenance of an online representation, which may also be necessary for processing complex objects; see Biederman, 1987; Marr \& Nishihara, 1978). Memory of those elements is, therefore, critical even when all the information necessary for successful performance is available in the display and no delays are imposed by the experimenter, and the MTL is a likely neural substrate for that memory.

In support of this theoretical role, reports have indicated that MTL damage causes impaired performance in discrimination paradigms that simultaneously present all stimuli, including odd-man-out tasks requiring identification of the object that does not belong with the others (Barense et al., 2007; Lee, Buckley, et al., 2005; Lee, Bussey, et al., 2005). Deficits in such paradigms occur in the absence of any imposed delays, and the originating authors have suggested that MTL structures play a critical role in perception and not just in memory (Graham, Barense, \& Lee, 2010). But, in light of the above considerations, we would argue that the absence of imposed delays does not rule out mnemonic contributions to ongoing processing (see also Shrager et al., 2008). In the odd-man-out paradigm, although all the objects in a given trial are available for inspection at all times, the size and complexity of the objects evoking deficient performance ensure that processing entails the serial inspection of each separate object and multiple comparisons among them while searching for some features that discriminate one from the rest (see also Lee \& Rudebeck, 2010). Each object, upon being fixated, must be compared with representations being held on-line of the other objects, necessitating the use of on-line representations despite the absence of any imposed delays. One means of evaluating whether patients with MTL damage can maintain information normally during such tasks would be to index the quality of their maintained representations at multiple time points.

To evaluate the role of MTL in on-line representations, we used a paradigm in which it was possible to examine the memory representations guiding performance and to assess any changes over brief intervals during on-line processing. Patients with MTL damage and healthy comparison subjects (henceforth, "MTL patients" and "comparison subjects," respectively) were tested on a complex visual search task. On each trial, they saw a display with a centrally placed

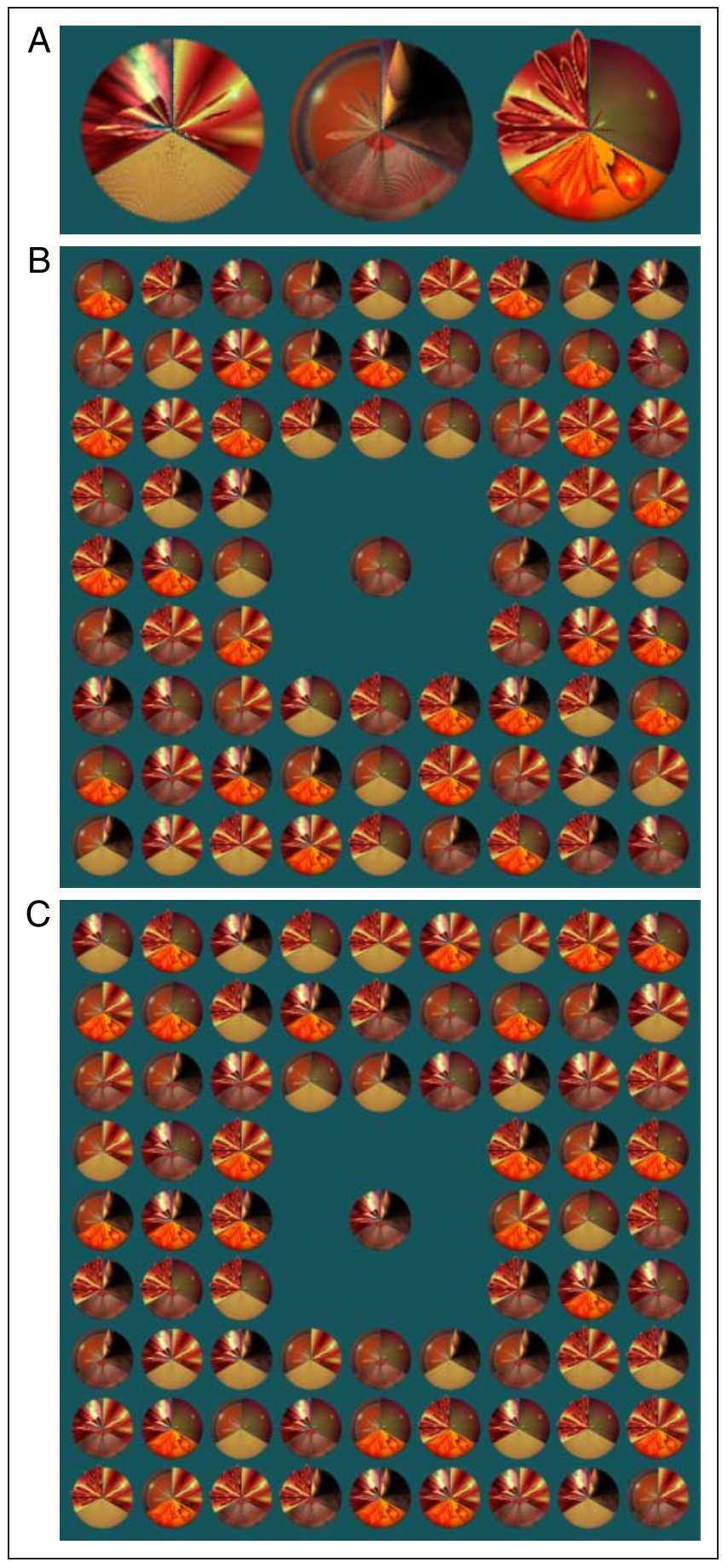

Figure 1. Illustrations of selected items and sample displays. (A) Three sample items illustrating the three-wedge design of all targets and lures and the three components that occurred in the top left, top right, and bottom portions of the items. (B and $\mathrm{C}$ ) Illustrations of target-present and target-absent test displays, respectively. Subjects searched each test display for an item that matched the central sample, responding "Yes" if they found a target and "No" if they did not. In $\mathrm{B}$, the target is located in Row 2 Column 7.

sample stimulus and 72 peripherally arranged stimuli, all of which remained visible throughout the trial (see Figure 1). The task was to indicate whether any of the peripheral stimuli matched the sample exactly. On one third of the 
trials, there was one exact match (i.e., the target), whereas the remaining stimuli were lures, differing from the target by varying degrees. Target-absent trials contained only lures. Subjects were able to visually inspect any peripheral item and refixate the sample item as often as they wished, until making their behavioral response. Eye movements recorded throughout each trial afforded a highly sensitive index of the representations being used to guide performance.

Three measures, one behavioral and two based on eye movements, provided critical insights. First, MTL patient task performance was above chance but still impaired despite the lack of imposed delays. Second, the duration of fixations to peripheral lure stimuli was found to be a function of the similarity of any given lure to the sample, with longer fixations to lures bearing greater resemblance to the sample, revealing the quality of the maintained representation (also see Warren, Duff, Tranel, \& Cohen, 2010). MTL patients exhibited the same function relating fixation duration to lure sample similarity as comparison subjects, but only when a modest number of fixations intervened between the last viewing of the sample item and fixating a given lure. As the number of intervening fixations increased, the modulation of fixation duration by lure sample similarity was attenuated in MTL patients relative to the comparison subjects. Third, all subjects frequently refixated the sample, providing them with an opportunity to refresh their representation before sampling further peripheral stimuli. However, over trials, comparison subjects gradually returned to the sample at fewer times per trial and fixated more peripheral stimuli before returning to the sample, whereas MTL patients continued to refresh their representations by fixating the sample at a constant rate across trials. Taken together, these three findings reveal the more rapid degradation of MTL patients' representations and greater dependence on refreshing their representations during on-line processing of complex displays, along with impaired behavioral performance, despite all information needed to succeed being readily available in the display and the absence of any experimenter-imposed delays. These findings indicate that the MTL participates in the active construction of novel representations even during a superficially non-mnemonic task.

\section{METHODS}

\section{Subjects}

Five amnesic patients with extensive bilateral hippocampal damage participated in the experiment, all of whom were drawn from the Patient Registry of the Division of Behavioral Neurology and Cognitive Neuroscience at the University of Iowa. Three of the patients were amnesic secondary to an anoxic episode (1606, 1846, and 2363), and the MTL damage in these patients was relatively circumscribed; atrophy of bilateral hippocampus has been reported for each individual (Allen, Tranel, Bruss, \& Damasio, 2006). Two of the patients were amnesic secondary to HSE (1951, 2308), and both exhibited lesions including hippocampus bilaterally and extending well into the temporal lobes (for details of 1951, see Feinstein et al., 2009). Damage to extrahippocampal MTL structures was quite severe in both patients, and the volume of the remaining tissue was substantially less than normal in both when it could be identified and measured at all. Detailed neuropsychological and demographic information for the participating patients is available in Table 1. Five healthy comparison subjects recruited from the Champaign-Urbana community also participated, each matched to a participating patient on sex, age, years of education, and handedness. All subjects granted informed consent before their experimental session.

\section{Apparatus}

All stimulus displays were presented on an NEC MultiSyncLCD2190UXi LCD monitor with a diagonal measurement of $54 \mathrm{~cm}$, a native resolution of $1600 \times 1200$ pixels, and a vertical refresh rate of $60 \mathrm{~Hz}$. The eye-tracking apparatus was an Applied Sciences Laboratory R6/VHT-2

Table 1. Demographic and Neuropsychological Description of MTL Patients

\begin{tabular}{|c|c|c|c|c|c|c|c|c|c|}
\hline \multirow[b]{2}{*}{ Patient ID } & \multirow[b]{2}{*}{ Etiology } & \multirow[b]{2}{*}{ Sex } & \multirow[b]{2}{*}{ Hand } & \multirow[b]{2}{*}{ Onset } & \multirow[b]{2}{*}{ Age } & \multirow[b]{2}{*}{$E d$} & \multirow{2}{*}{$\frac{\text { WAIS }}{\text { FSIQ }}$} & \multicolumn{2}{|c|}{ WMS } \\
\hline & & & & & & & & GMI & $D R I$ \\
\hline 1606 & Anoxic & M & $\mathrm{R}$ & 1990 & 61 & 12 & 91 & 66 & 61 \\
\hline 1846 & Anoxia & $\mathrm{F}$ & $\mathrm{R}$ & 1993 & 45 & 14 & 84 & 57 & 62 \\
\hline 1951 & HSE & M & $\mathrm{R}$ & 1980 & 56 & 16 & 121 & 75 & 53 \\
\hline 2308 & HSE & $\mathrm{M}$ & $\mathrm{L}$ & 1999 & 52 & 16 & $98^{\mathrm{a}}$ & 45 & 48 \\
\hline 2363 & Anoxia & $\mathrm{M}$ & $\mathrm{R}$ & 1998 & 52 & 16 & 198 & 73 & 74 \\
\hline
\end{tabular}

Neuropsychological and demographic details of participating neurological patients. WAIS $=$ Wechsler (1997) adult intelligence scale-III, FIQ $=$ fullscale IQ, WMS = Wechsler memory scale-III, GMI = general memory index, DRI = delayed recall index. For the WMS, the DRI is an average of the auditory delayed index and visual delayed index. All tests yield mean scores in the normal population of 100 with an $S D$ of 15 .

${ }^{a}$ Reflects a re-evaluated FSIQ for this participant. 
remote eye-tracking system with a video-based headtracking system for enhanced stability; the system sampled point of gaze at a rate of $60 \mathrm{~Hz}$.

\section{Stimulus Materials}

This experiment employed novel visual stimuli composed of three segments each. Each segment was a wedge, resembling one third of a circle, and when presented in the correct configuration, three segments comprised a roughly circular stimulus item (samples are provided in Figure 1A). Stimuli were approximately 115 pixels and $2.3^{\circ}$ of visual angle in diameter.

\section{Procedure}

The subject was seated at a comfortable viewing distance from the monitor, approximately $75 \mathrm{~cm}$. During each trial, the subject performed a visual search task with no time limit. After searching an array for the target item, the subject responded "Yes" (i.e., target present) or "No" (i.e., target absent); responses were either made verbally or by button press per individual preference.

Lure items and sometimes a target item filled much of the screen. Specifically, the screen displayed a $9 \times 9$ grid centered on the sample item (see Figure 1B and C). Only the eight spaces adjacent to the sample were unfilled, leaving 72 peripheral items. Center-to-center, adjacent stimuli in the array were separated by at least $2.6^{\circ}$ of visual angle. Peripheral items varied in the number of segments that matched the sample item's segments, and in a target-absent trial: 24 items had no such segments, 24 more items had one such segment, and the remaining 24 items had two such segments. During targetpresent trials, the target item randomly replaced one lure item, and the only constraint on its position was the location of the preceding trial's target item (if any occurred); that location and nearby locations were ruled out.

Eighty-one trials were completed, 27 containing targets (Figure 1B and C). Target incidence was constrained so that the first trial did not contain a target item, no more than two trials in a row contained a target item, and each third of the experiment contained nine target-present trials but was otherwise random. Positioning of items within the peripheral array was constrained to enforce an approximately equal density of zero-, one-, and two-match lures throughout but was otherwise random. Twenty-seven unique sample items were used three times each, once in each third of the experiment (i.e., 27 of 81 trials).

Eye movement data were collected while the task was being performed, and a calibration screen was presented before each trial to ensure that point of gaze was being gauged accurately. Trial phase was advanced from the intervening calibration screens by the experimenter while central fixation was maintained. During test trials, free viewing appropriate to search was encouraged.

\section{Eye Movement Data}

Fixation data were generated from $60-\mathrm{Hz}$ samples of point of gaze using Applied Science Laboratories' Eyenal application (version 2.91), and this process considered raw eye movements falling within $0.5^{\circ}$ during intervals of $83 \mathrm{msec}$ or greater to be fixations. These fixation data and the transitions between them were analyzed according to generally accepted principles (e.g., Hannula et al., 2010), and some specifics are reported below.

In our task, the positions of a subject's fixations were recorded and later binned into predetermined ROIs containing one stimulus item each. ROIs for the peripheral items were necessarily small owing to the dense layout, but the central sample item was granted a larger ROI because of its isolation and its importance for many of the analyses presented here. Each ROI was square and centered on its corresponding item. Peripheral ROIs were 130 pixels square $\left(\sim 2.5^{\circ}\right.$ visual angle $)$, and the central sample ROI was 260 pixels square $\left(\sim 5^{\circ}\right.$ visual angle). The ROI and duration of each fixation were coded, and transitions between ROIs were also noted.

Eye movement data were included in the set to be analyzed only if they exceeded an objective criterion for eye-tracking quality. All trials included in further analyses had fixation data accounting for at least $50 \%$ of the trial duration (i.e., elapsed time from display onset until subject response). Trials with a lesser proportion of fixation data were considered unlikely to contain accurate measures of gaze position. Across all reported subjects, 13 of 810 trials were excluded on the basis of this objective quality criterion.

One important concern when testing severely amnesic patients using a complex visual search task was whether their memory deficit would allow them to complete a search of the entire array. Before beginning any other analyses, we evaluated this question both qualitatively and quantitatively. On the basis of those evaluations (described in the Supplementary Materials), we were satisfied that MTL patients searched the arrays as thoroughly as comparison subjects.

\section{Statistical Analyses}

All statistical analyses were conducted in the $\mathrm{R}$ (version 2.12.2), SAS (version 9.2), or Python (version 2.7) programming languages. Data were subject to one or two levels of inferential analysis: (1) $t$ tests or ANOVAs as appropriate, using the mean observed values of each subject as the dependent variables; and (2) model-based inference using generalized hierarchical linear models (GHLM). For the first level, test results noted in the Results section indicate evaluation of the group-level differences on the basis of only per-subject mean values, whereas in the second case, Type III fixed effects tests indicate the reliability of parameter values associated with predictors in models that used each datum as an outcome. 


\section{Behavioral}

Accuracy in detecting a target among lures was summarized using $d^{\prime}$ to control for potential criterion differences between subjects. Calculation employed a method that adjusts for ceiling and floor performance when necessary (Macmillan \& Creelman, 2005). Analysis of behavioral performance using proportion correct was congruent with the reported $d^{\prime}$ analyses.

Group-level differences in RT across the entire experiment were evaluated using $t$ tests of per-subject averages of RT during correct rejection trials. RT changes over time during the experiment were analyzed using a GHLM implemented with the GLIMMIX SAS procedure: Predictors included were MTL status, ordinal value of test trials, and an interaction term; subjects were treated as a random effect to account for individual variation in RT; RT was assumed to follow a log-linked gamma distribution; ordinal value of test trial was treated as a continuous predictor; and MTL damage was a discrete predictor with two levels.

\section{Eye Movement}

Fixation duration. Data from correctly rejected targetabsent trials included 48,736 fixations, and the distribution of fixation durations had several general properties that directed the analyses we conducted. Observed minimum fixation duration was $83 \mathrm{msec}$ (i.e., our enforced minimum value), maximum fixation duration was $2085 \mathrm{msec}$, and visual inspection of the distribution revealed pronounced positive skew. A log transform was applied to fixation duration data before any averaging to address the positive skew, and our plotted data reflect that transformation. Initial evaluation of fixation duration data, therefore, involved first log-transforming the raw values and then calculating persubject, per-condition means as the outcome variable for a $3 \times 2$ repeated measures ANOVA that included predictors for the number of features that a lure shared with the target (i.e., 0, 1, or 2), MTL status (i.e., damaged or healthy), and an interaction term. Next, to investigate whether the observed fixation duration difference varied over time between groups, we introduced a viewing latency predictor to indicate whether a given fixation was made early or late in search (i.e., within six fixations since sample fixation versus seven fixations or later). This analysis was inspired by visual inspection of the group mean data, as was the selection of the criterion (see Supplementary Materials for details). We chose to characterize latency as a binary value to avoid unwarranted assumptions about a specific relationship between the ordinal value of latency and fixation durations and also because it provides a convenient shorthand. Once again, we averaged logtransformed per-subject, per-condition fixation durations, then subtracted the mean fixation durations for zero-match lures from those for two-match lures, creating a difference score for each subject corresponding to the cells of a $2 \times 2$ repeated measures ANOVA, with factors of MTL status, viewing latency, and an interaction term. Retaining data relevant to only zero- and two-match lures for this second analysis left 33,768 fixations.

Transitions to sample. Changes to transitions to the sample item per correct rejection trial over time were analyzed with a GHLM including fixed effects for trial as an ordinal value and MTL status as a two-level factor; unique subjects were entered as a normally distributed random effect. As the outcome variable consisted of counts, its distribution was assumed to be negative binomial. The best-fit model, reported below, employed both predictor variables and an interaction between them.

Maximum search path length. Two predictor variables were used to model any changes in the maximum search path lengths observed during correct rejection trials across trials. Ordinal value of trial was treated as a continuous predictor, and MTL status was employed as a discrete predictor. As the outcome variable consisted of counts, its distribution was assumed to be negative binomial. The best-fit model, reported below, employed both predictor variables and an interaction between them.

\section{RESULTS}

\section{Behavioral Performance}

Subjects made a yes-no judgment on each trial, indicating whether they thought the display contained a target exactly matching the sample. Analysis of behavioral responses using $d^{\prime}$ as an index of accuracy indicated that MTL patients performed better than chance responding would have allowed $\left(T_{4}=6.263, p=.003\right)$, but they were impaired relative to comparison subjects $\left(T_{8}=3.640, p=.007\right)$ with all patients scoring below the range of comparison subjects' performance (see Figure 2A). Incorrect responses by patients were most often misses (see Table 2). Etiology and extent of MTL damage were not reliably related to any aspect of behavioral performance; although anoxic patients all had substantially less temporal lobe tissue loss than HSE patients, the performance of the two groups was mixed and indistinguishable (see Figure 2A). Performance was not correlated with either of two general measures of memory performance (see Table 1), the Wechsler Memory Scale Third Edition (Wechsler, 1997) General Memory Index $(r=-0.087, p=.889)$ or its Delayed Recall Index $(r=-0.161, p=.796)$.

Subjects were asked to perform their search with both speed and accuracy, but displays were presented until a response was made. Initial analysis of RTs for correctly rejected (target-absent) test trials (see Figure 2B) suggested that MTL patients were marginally slower than comparison subjects $\left(T_{8}=2.181, p=.061\right)$. We investigated both that difference and changes to RT during the experiment in more detail using a GHLM, which also suggested that 
Figure 2. Summary of the behavioral data. (A) MTL patients were reliably impaired at search relative to comparisons, and their scores are individually labeled for reference. (B) All subjects searched more quickly as the experiment continued, although patients were slower than comparisons overall. Individual RTs and best-fit regression lines are shown.
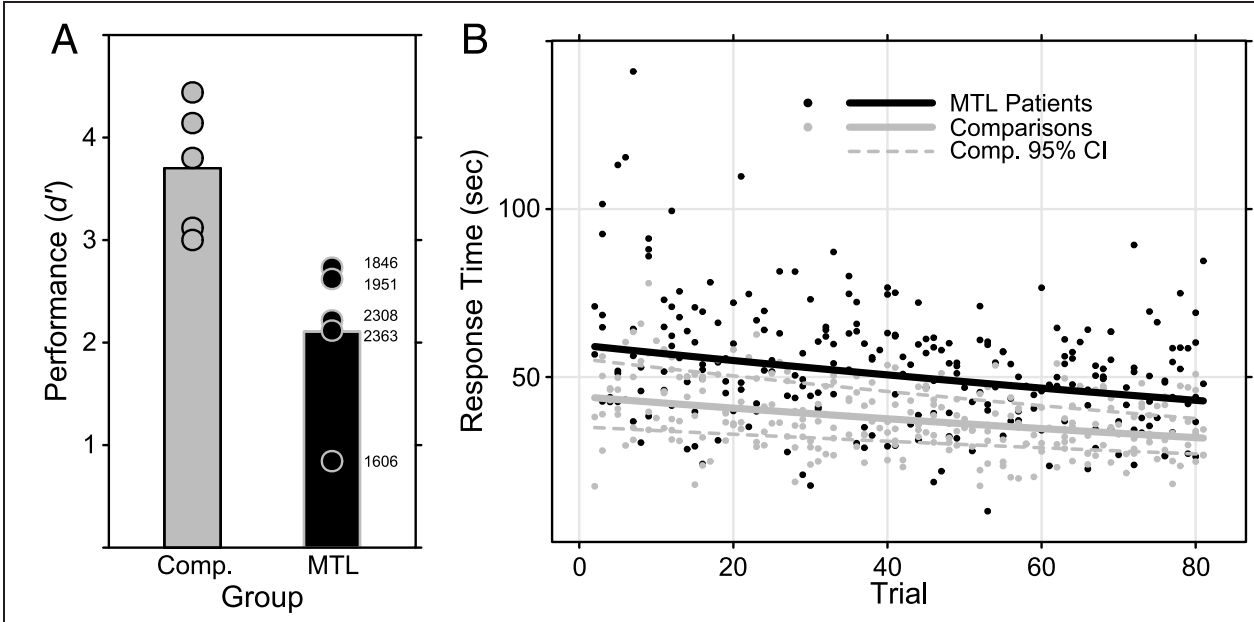

overall MTL patients were slower than comparison subjects in responding (here reliably; $T_{495}=2.230, p=$ .026) but indicated that both subject groups showed decreased RT across trials $\left(T_{495}=6.820, p<.0001\right)$, whereas the lack of a significant interaction suggested that both groups benefitted from repetition at the same rate.

\section{Eye Movement Measures}

Subjects' eye movements were recorded during all trials, and several measures of eye movement behavior were extracted for each viewer. Two sets of measures were of primary importance:

One concerned the duration of fixations to the various peripheral items as a function of each item's similarity to the central sample. Items varied in having from zero to three segments in common with the sample (lures had 0-2 segments matching the sample; a target-present trial's single target stimulus was a 3-segment match; see Figure 1A-C). To the extent that the duration of fixations to lures was positively related to similarity to (i.e., the number of segments they shared with) the sample, the measure provided an index of the quality or integrity of the on-line representation of the sample.

The other concerned the pattern of viewing of the sample item. During the course of each trial, subjects were able to return their gaze to the sample item as often as they liked, which they tended to do interspersed among their search of the peripheral stimuli. We examined the number of times each subject transitioned back to the sample item from anywhere among the peripheral stimuli as well as the length of the search paths (i.e., the number of fixations to peripheral stimuli before returning to the central sample) on each trial. These measures of viewing directed at the sample provide an index of subjects' need to refresh their representation of the sample so as to guide their search of the display.

\section{Fixation Durations}

Lure items were fixated by subjects for varying amounts of time, and that variation was reliably different for lure items sharing different numbers of segments with the target $\left(F_{2,16}=152.787, p<.0001\right)$, and no difference was evident between groups $\left(F_{1,8}=0.989, p=.349\right)$ or in the fixation duration effect between groups $\left(F_{2,16}=1.402, p=\right.$ .275). All pairwise differences between levels of shared segments were reliable (maximum Bonferroni-corrected $p=$ .0168; see Figure 3A). The same fixation duration data were next examined more closely for evidence of group-level differences during search by calculating the mean difference in durations between fixations to zero- and two-match lures and by introducing a factor indicating whether a fixation occurred early or late in search (i.e., within six or fewer fixations since the sample was last fixated vs. seven or more fixations; see Methods and Supplementary Materials for details). The interaction of MTL status by fixation latency was reliable $\left(F_{1,8}=6.471, p=.0345\right)$, whereas neither

Table 2. Participant Performance and RTs

\begin{tabular}{|c|c|c|c|c|c|}
\hline \multirow[b]{2}{*}{$I D$} & \multirow[b]{2}{*}{ Group } & \multicolumn{2}{|c|}{ Hits } & \multicolumn{2}{|c|}{ CRs } \\
\hline & & Rate & $R T(\mathrm{sec})$ & Rate & $R T(\mathrm{sec})$ \\
\hline 1606 & MTL & 0.556 & 19.597 & 0.759 & 30.959 \\
\hline 1846 & MTL & 0.741 & 24.244 & 0.981 & 52.073 \\
\hline 1951 & MTL & 0.704 & 26.857 & 0.981 & 59.359 \\
\hline 2308 & MTL & 0.667 & 46.612 & 0.963 & 57.457 \\
\hline 2363 & MTL & 0.630 & 28.911 & 0.963 & 60.311 \\
\hline $1606 c$ & Comp & 0.963 & 29.036 & 1.000 & 40.799 \\
\hline $1846 c$ & Comp & 1.000 & 25.714 & 1.000 & 44.037 \\
\hline 1951c & Comp & 0.778 & 18.789 & 1.000 & 28.431 \\
\hline $2308 c$ & Comp & 0.926 & 26.932 & 1.000 & 45.861 \\
\hline $2363 c$ & Comp & 0.741 & 19.556 & 1.000 & 34.137 \\
\hline
\end{tabular}

Per-subject hit and correction-rejection rates and mean RTs. Patients were much more likely to miss stimuli than comparisons, but both groups correctly rejected most target-absent displays. Patients were also marginally slower than comparisons overall. Statistical analyses of both measures are available in the Results section. 
MTL status nor fixation latency alone were reliable (respectively: $F_{1,8}=2.718, p=.138 ; F_{1,8}=0.961, p=.356$ ). Post hoc tests confirmed that MTL patients showed less difference in fixation durations in late search than early search (paired $T_{4}=3.209, p=.033$ ), whereas healthy comparison subjects did not differ across latency (paired $T_{4}=0.935, p=$ .403). This suggests that both groups maintained a good representation of the sample early in search, but that representation was maintained better by comparison subjects while searching (Figure 3B). Notably, the same interaction was reliable both in the fixation data from the entire experiment and from data collected within correct rejection trials during the first third of the experiment before any sample targets were repeated $\left(F_{1,8}=8.904, p=.0175\right)$, and the same pattern of decreased fixation duration differences for patients at longer search latencies is evident (Figure 3C). Even when analysis was limited to correct rejections during the first nine trials of the experiment, the same effects are evident $\left(F_{1,8}=9.861, p=.0138\right)$, suggesting that limited experience of the stimulus materials still permits the effect to be observed.

\section{Transitions to Sample}

Fixations to the central sample item were frequent for all subjects in early trials; given the nature of the task and the continuous availability of the sample, this was not surprising. Overall, MTL patients made marginally more fixations to the central sample item than comparison subjects during correct rejection trials $\left(T_{8}=2.170, p=.062\right)$. However, over the course of the experiment, there were numerical changes in the number of transitions to the sample for comparison subjects that were not apparent for patients. A regression model indicated that later trials were associated with fewer transitions to the sample item for all subjects $\left(T_{495}=11.060, p<.0001\right)$, but a reliable interaction with MTL status $\left(T_{495}=6.570, p<.0001\right)$ indicated that this effect was stronger in comparison subjects and attenuated in patients (see Figure 4A). There was no main effect of MTL status $\left(T_{495}=1.31, p=.192\right)$.

\section{Longest Fixation Path}

The search paths of MTL patients were shorter than those of comparison subjects $\left(T_{8}=2.770, p=.024\right)$. Paralleling the preceding analysis, the greatest number of fixations a given subject made per trial before returning to the sample was also modeled, and again differences between patients and comparison subjects were observed. Later trials were associated with increasing path length in comparison subjects $\left(T_{495}=9.000, p<.0001\right)$, and the interaction between MTL damage and trial was also reliable $\left(T_{495}=\right.$ $6.670, p<.0001)$. That interaction indicated that patients did not increase the length of their search paths from the sample item across the experiment (see Figure 4B). There was no main effect of MTL status according to this model $\left(T_{495}=1.34, p=.183\right)$.

Taken together, these analyses of fixation path length and transitions to sample indicate that comparison subjects were able to exploit knowledge about the search array items in ways that patients were not. Specifically, despite repeated mass exposure to the set of items, patients did not change their search patterns much if at all. In the context of the fixation duration data, it seems likely that
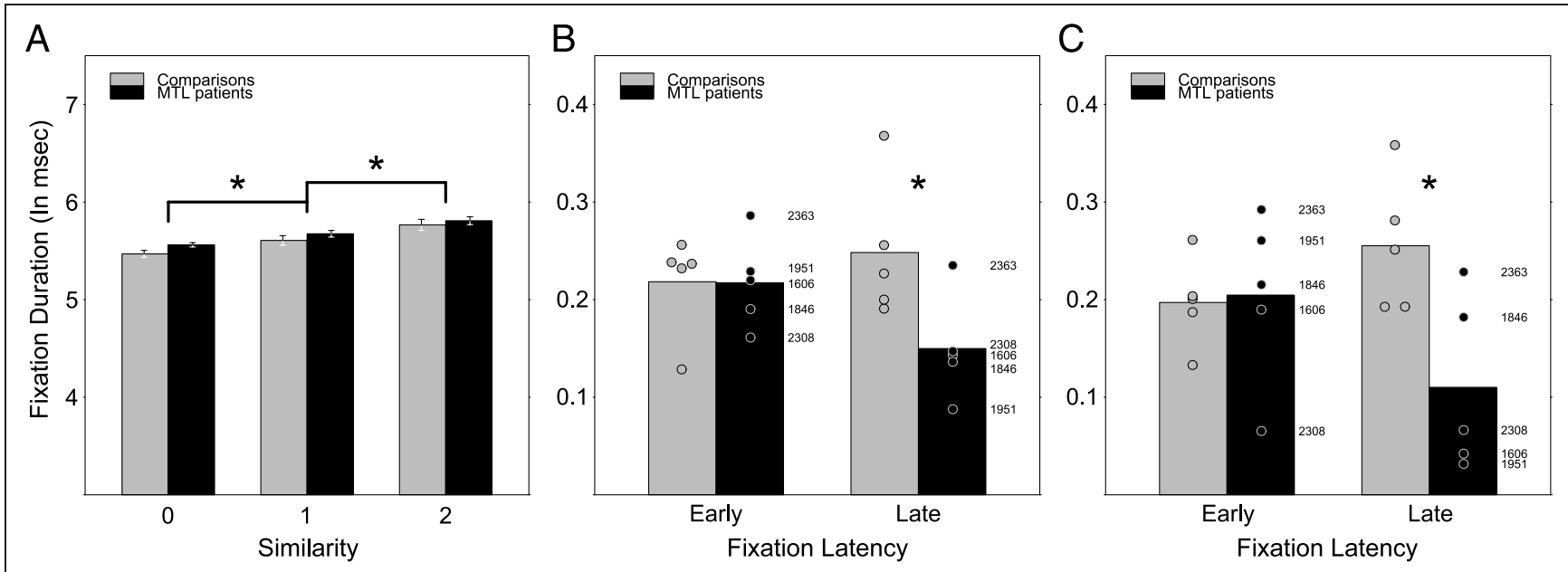

Figure 3. Modulation of fixation duration by lure sample similarity. Note that the $y$ axes of all three panels use ln (msec) units, but that A uses a different scale than $\mathrm{B}$ and $\mathrm{C}$, which both plot differences in fixation durations rather than whole fixation durations. (A) Both comparisons and MTL patients fixated items that resembled the sample for longer than those that did not. Whiskers indicate SEM. (B and C) However, as more fixations intervened between the last viewing of the sample item and the fixation of a given lure, this effect was attenuated in patients and exaggerated in comparisons; these plots illustrate the effect using the difference in fixation durations between two- and zero-match lures early and late in search. Group means are presented as bars whereas the difference observed for each subject is plotted as a point, and patient values are individually labeled for reference. B summarizes data from the entire experiment, whereas $\mathrm{C}$ summarizes data from only the first third of the experiment (i.e., 27 trials). Asterisk indicates reliable differences at $p<.05$ : differences between levels of lure sample similarity in A and reliable MTL status by latency interactions in B and C. 
Figure 4. Evidence of learning across the course of the experiment as reflected in eye movement measures. Both plots show individual values and best-fit regression functions. (A) Comparisons fixated the sample item less and less frequently as the experiment continued, whereas MTL patients continued fixating the sample item at approximately the same rate throughout. (B) Likewise, comparisons made longer and longer forays from the sample item across the course of the experiment, fixating more items before returning. The lengths of patients' search paths were stable across the entire experiment.

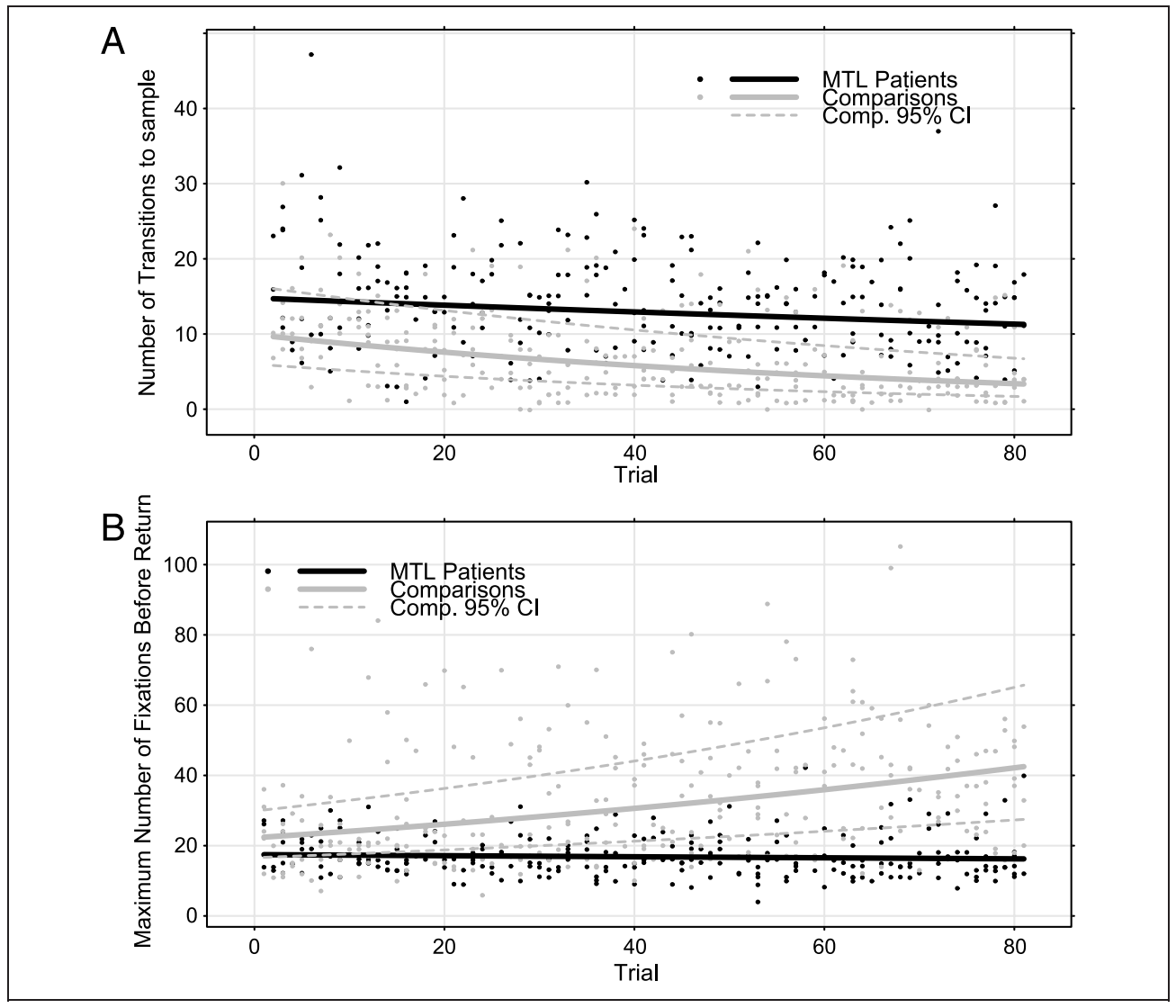

patients were intentionally limiting the length of their search paths to preserve some degree of performance, suggesting some adaptation to failures of on-line representation.

\section{DISCUSSION}

The current findings indicate that MTL structures aid in the maintenance or construction of on-line representations used during ongoing processing. Patients with damage to the MTL were impaired when performing a task that had no imposed delays, that is, when all information necessary for successful performance was available in the display. Moreover, eye movement measures sensitive to the quality of viewers' on-line representations and to the use of those representations in guiding ongoing task performance showed abnormally rapid degradation of such representations in MTL patients, and the behavioral deficits seen here did not require MTL damage outside the hippocampus. Consequently, the performance of MTL patients in this experiment permits inference about the time course of visual representations supported by structures outside the MTL.

A critical measure here was the duration of fixations to lure stimuli, which varied systematically with lure sample similarity for all subjects (see also Warren et al., 2010), providing us with an index of the on-line representations used by viewers. MTL patients showed a reduced modulation of fixation duration by lure sample similarity during long fixa- tion paths away from the sample, revealing directly the degradation of their on-line representations. Abnormally rapid changes in the nature or quality of these representations in the absence of hippocampus could be caused by a variety of mechanisms. Temporal decay is one possibility, and evidence supporting this explanation has been observed in MTL patients tested with simple visual stimuli (Warren et al., 2010; Olson, Moore, et al., 2006; Sidman, Stoddard, \& Mohr, 1968; Prisko, 1963). One previous report (Warren et al., 2010) demonstrated that when MTL patients performed a much simpler visual search task, fixation duration effects were normal when a sample target was available during search, but the same fixation duration effects were abnormal if a 6-sec delay was imposed between sample presentation and search. However, delays in all of the cited reports were always unfilled, whereas the current task involved ongoing search while the sample representation was maintained. Rapid sequential fixation of multiple lure items might interfere with the sample target's representation, weakening or distorting it. Follow-up experiments could adapt our paradigm to more tightly control search experience (i.e., search history) while preserving elements of organic search behavior, and this approach would allow temporal decay and interference to be convincingly disentangled. These experiments would also address the underlying relationship between latency and representational integrity, which we characterized as binary 
(i.e., early or late) for convenience, but could reasonably be linear, binary, or still more complex.

Despite their impairment in search performance and in certain measures of eye movement behavior, MTL patients did exhibit some learning over time that was similar to healthy comparison subjects (i.e., faster RTs), and this can be attributed to intact nondeclarative learning mechanisms that have been demonstrated in many other tasks (e.g., Cavaco, Anderson, Allen, Castro-Caldas, \& Damasio, 2004; Brooks \& Baddeley, 1976; Milner, 1962). One prominent investigation of visual search task behavior in amnesic patients (Chun \& Phelps, 1999) employed a widely used, relatively simple variant (i.e., searching for $\mathrm{L}$ among Ts) to test contextual, relational learning. Although subjects performed many trials of the task, half of the search arrays were repeated many times without any forewarning. As in the current study, both amnesic patients and comparison subjects showed learning about the task by some measures (e.g., completing searches more quickly), but in that experiment, only comparison subjects exhibited a further enhancement of response speed associated with the repetition of specific search arrays. This pattern of sparing and impairment in search behavior is generally congruent with our results, although our more complex stimuli and task taxed patients in different ways and yielded different behaviors. In the Chun and Phelps experiment, maintaining a simple, consistent search instruction (i.e., find the L) presented no difficulty for patients across many trials, whereas in our task the more complex representations of targets that varied across trials proved more difficult, and our fixation duration analyses indicate that even while searching within a trial patients had difficulty maintaining the sample target representation.

MTL patients have been shown to perform poorly in other tasks involving judgments about complex visual stimuli (Barense et al., 2007; Lee, Buckley, et al., 2005; Lee, Bussey, et al., 2005), but the current results are unique in several important respects. First, our design allowed us to assess the quality of the representations supporting task performance and their degradation in MTL patients in an unprecedented manner, that is, by providing a sample of the target throughout each test trial and using parametric variation of lure sample similarity and observation of eye movements. Second, we observed impairment in MTL patients regardless of whether the damage was MTL-wide or was more focally hippocampal. Lesions limited to the hippocampus have not been reported previously to impair the discrimination of complex objects, although damage to that structure has been associated with impairments in scene discrimination (Lee, Buckley, et al., 2005; Lee, Bussey, et al., 2005). Third, although it has been suggested that similar impairments in amnesic performance solely reflect dysfunction of a well-characterized long-term memory system (Shrager et al., 2008), our results demonstrate that irrespective of the underlying mechanisms, apparent attempts by MTL patients to adjust their behavior to compensate for their impairment did not succeed completely.
Even with all necessary material available for indefinite inspection, their performance was reliably impaired.

Neuropsychological investigation has revealed deficits in short-delay performance by MTL patients (e.g., Ezzyat \& Olson, 2008; Shrager et al., 2008; Barense et al., 2005, 2007; Hannula et al., 2006, 2007; Hartley et al., 2007; Nichols et al., 2006; Olson, Moore, et al., 2006; Lee, Buckley, et al., 2005; Lee, Bussey, et al., 2005; Holdstock et al., 2002; Ryan et al., 2000; Buffalo et al., 1998), but it is only one of several converging methods pointing to a role for the MTL in maintenance or integration of stimulus materials. Functional neuroimaging of healthy participants has also contributed substantially to this field (e.g., Olsen et al., 2009; Hannula \& Ranganath, 2008; Ranganath et al., 2005; Ranganath, Cohen, Dam, \& D'Esposito, 2004; Ranganath \& D'Esposito, 2001; Stern, Sherman, Kirchhoff, $\&$ Hasselmo, 2001) by demonstrating that MTL regions are particularly active while information is maintained. One notable example (Olsen et al., 2009) required participants to perform a delayed-match-to-sample task using a pair of faces as the stimuli to be maintained. High-resolution functional images indicated that two MTL regions (entorhinal and perirhinal cortex along with anterior hippocampus) showed continuous activation throughout the delay period when a correct response would be made immediately afterward, whereas other MTL regions (parahippocampal and fusiform cortex) responded in an anticipatory fashion, simply showing more activation as the test phase approached irrespective of the outcome. This pattern of results might suggest that information is actively maintained in MTL regions during delay intervals, although other reports of MTL activations predicting subsequent memory after delays of seconds suggest that continuous activation of these structures may not be necessary (e.g., Hannula \& Ranganath, 2008). In either case, the relationship between MTL activation and performance across short intervals appears to be robust across several experimental paradigms and approaches.

The results reported here suggest that damage to the MTL results in visual search deficits that are principally mnemonic in nature, and this explanation can address many other findings of behavioral impairment in MTL patients. We identify these deficits as mnemonic because MTL patients show normal fixation of lures at short latencies, suggesting that there is an early representation of the sample item, and therefore structures outside the MTL must be sufficient to briefly maintain that representation. Likely candidates for this early maintenance include portions of prefrontal and parietal cortex, potentially engaged in cooperative interactions: Portions of $\mathrm{pFC}$ have been associated with executive control processes and enhancement of maintenance across delays (Zanto, Rubens, Thangavel, \& Gazzaley, 2011; Rossi, Pessoa, Desimone, \& Ungerleider, 2009; Gazzaley, Rissman, \& D'Esposito, 2004; Barcelo, Suwazono, \& Knight, 2000); and parietal cortex has been implicated in visual working memory using a wide variety of tests and cognitive neuroscience methods (Berryhill \& 
Olson, 2008b; e.g., Koenigs, Barbey, Postle, \& Grafman, 2009; Berryhill \& Olson, 2008a; see also Wager \& Smith, 2003). If these regions are sufficient to maintain a representation briefly, then the MTL (and perhaps the hippocampus in particular) may enhance the resilience of the representation, making it less susceptible to interference or degradation over time, although whether any enhanced resiliency is necessarily tied to long-term representation (e.g., Shrager et al., 2008) remains an open question. Meanwhile, if the patients had defective perception of complex items (cf. Graham et al., 2010), then presumably these effects would not manifest normally regardless of whether viewing took place earlier or later. Instead, it seems that a normal (or at least sufficient) representation is formed initially but that it is degraded or disrupted by time or interference. Our results leave open the possibility that the representation initially formed by patients may be different in kind from those formed by comparison subjects, even if that representation is initially similar enough to drive normal eye movement effects. A lack of rapid, durable pattern separation between the sample and lure items under consideration might explain the evanescence of the representations that patients can form, and certain theories of MTL function predict poor pattern separation after hippocampal damage (Norman, 2010; Norman \& O'Reilly, 2003).

Relational memory theory (Eichenbaum \& Cohen, 2001; Cohen \& Eichenbaum, 1993) may also provide a useful framework for interpreting the current results, as it already addresses a variety of deficits caused by hippocampal damage (Konkel, Warren, Duff, Tranel, \& Cohen, 2008; Hannula et al., 2007; Ryan \& Cohen, 2004a; Ryan et al., 2000). In the course of performing our search task, subjects needed to maintain a representation of the sample stimulus while exploring many similar items, and one conceptualization of this process is that the maintained representation is associated or related to a distinct, abstract concept of "goal" or "target." If the association between sample representation and the "goal" concept was disrupted, search could not be successful, and a potential disruption would have been the accidental substitution of a lure representation (i.e., an item previously considered during search) for the sample representation, which would have produced a continuing search for the wrong item, at least until the sample was fixated again. Such a failure of relational memory binding in ongoing processing would concur with a recent report in which patients with bilateral hippocampal sclerosis were impaired in a task requiring maintenance of scenes that would later need to be discriminated from highly similar lures, performance for which was shown via magneto-encephalography to require strong coupling of theta frequency activity in the temporal and occipital lobes in healthy subjects during memory maintenance (Cashdollar et al., 2009).

Taken altogether, the current findings demonstrate that the contributions to memory of the hippocampus and related MTL structures extend beyond the formation of enduring memory representations that support later retrieval of memory for facts and events to include the rapid creation of representations that immediately influence ongoing cognition and behavior.

\section{Acknowledgments}

The authors would like to acknowledge the following funding sources: NINDS P01 NS19632 (D. T.) and NIMH RO1 MH062500 (N. J. C.). Additionally, the authors would like to thank Jacob Gibbons, Aashesh Verma, and Dr. Carolyn Anderson. D. E. W. and N. J. C. conceived the experiments. D. E. W. implemented and administered the experiments and analyzed data. D. T. and M. C. D. provided access and support for testing subjects with amnesia. All authors cowrote the article, discussed results, and commented on the manuscript.

Reprint requests should be sent to David E. Warren, Department of Neurology, University of Iowa College of Medicine, 200 Hawkins Drive, 2192 RCP, Iowa City, IA 52242, or via e-mail: davideugenewarren@gmail.com.

\section{REFERENCES}

Allen, J. S., Tranel, D., Bruss, J., \& Damasio, H. (2006). Correlations between regional brain volumes and memory performance in anoxia. Journal of Clinical and Experimental Neuropsychology, 28, 457-476.

Barcelo, F., Suwazono, S., \& Knight, R. T. (2000). Prefrontal modulation of visual processing in humans. Nature Neuroscience, 3, 399-403.

Barense, M. D., Bussey, T. J., Lee, A. C. H., Rogers, T. T., Davies, R. R., Saksida, L. M., et al. (2005). Functional specialization in the human medial-temporal lobe. Journal of Neuroscience, 25, 10239-10246.

Barense, M. D., Gaffan, D., \& Graham, K. S. (2007). The human medial-temporal lobe processes online representations of complex objects. Neuropsychologia, 45, 2963-2974.

Berryhill, M. E., \& Olson, I. R. (2008a). Is the posterior parietal lobe involved in working memory retrieval? Evidence from patients with bilateral parietal lobe damage. Neuropsychologia, 46, 1775-1786.

Berryhill, M. E., \& Olson, I. R. (2008b). The right parietal lobe is critical for visual working memory. Neuropsychologia, 46, 1767-1774.

Biederman, I. (1987). Recognition-by-components: A theory of human image understanding. Psychological Review, 94, 115-147.

Brooks, D. N., \& Baddeley, A. D. (1976). What can amnesic patients learn? Neuropsychologia, 14, 111-122.

Buffalo, E. A., Reber, P. J., \& Squire, L. R. (1998). The human perirhinal cortex and recognition memory. Hippocampus, 8, 330-339.

Cashdollar, N., Malecki, U., Rugg-Gunn, F. J., Duncan, J. S., Lavie, N., \& Duzel, E. (2009). Hippocampus-dependent and -independent theta-networks of active maintenance. Proceedings of the National Academy of Sciences, U.S.A., 106, 20493-20498.

Cavaco, S., Anderson, S. W., Allen, J. S., Castro-Caldas, A., \& Damasio, H. (2004). The scope of preserved procedural memory in amnesia. Brain: A Journal of Neurology, 127, 1853-1867.

Chun, M. M., \& Phelps, E. A. (1999). Memory deficits for implicit contextual information in amnesic subjects with hippocampal damage. Nature Neuroscience, 2, 844-847.

Cohen, N. J., \& Eichenbaum, H. (1993). Memory, amnesia and the hippocampal system. Cambridge, MA: MIT Press. 
Cohen, N. J., \& Squire, L. R. (1980). Preserved learning and retention of pattern-analyzing skill in amnesia-Dissociation of knowing how and knowing that. Science, 210, 207-210.

Eichenbaum, H., \& Cohen, N. J. (2001). From conditioning to conscious recollection: Memory systems of the brain. New York: Oxford University Press.

Ezzyat, Y., \& Olson, I. R. (2008). The medial-temporal lobe and visual working memory: Comparisons across tasks, delays, and visual similarity. Cognitive, Affective E Behavioral Neuroscience, 8, 32-40.

Feinstein, J. S., Rudrauf, D., Khalsa, S. S., Cassell, M. D., Bruss, J., Grabowski, T. J., et al. (2009). Bilateral limbic system destruction in man. Journal of Clinical and Experimental Neuropsychology, 32, 88-106.

Gazzaley, A., Rissman, J., \& D'Esposito, M. (2004). Functional connectivity during working memory maintenance. Cognitive, Affective E Behavioral Neuroscience, 4, 580-599.

Graham, K. S., Barense, M. D., \& Lee, A. C. (2010). Going beyond LTM in the MTL: A synthesis of neuropsychological and neuroimaging findings on the role of the medial-temporal lobe in memory and perception. Neuropsychologia, 48, 831-853.

Hannula, D. E., Althoff, R. R., Warren, D. E., Riggs, L., Cohen, N. J., \& Ryan, J. D. (2010). Worth a glance: Using eye movements to investigate the cognitive neuroscience of memory. Frontiers in Human Neuroscience, 4, 166.

Hannula, D. E., \& Ranganath, C. (2008). Medial-temporal lobe activity predicts successful relational memory binding. Journal of Neuroscience, 28, 116-124.

Hannula, D. E., Ryan, J. D., Tranel, D., \& Cohen, N. J. (2007) Rapid onset relational memory effects are evident in eye movement behavior, but not in hippocampal amnesia Journal of Cognitive Neuroscience, 19, 1690-1705.

Hannula, D. E., Tranel, D., \& Cohen, N. J. (2006). The long and the short of it: Relational memory impairments in amnesia, even at short lags. Journal of Neuroscience, 26, 8352-8359.

Hartley, T., Bird, C. M., Chan, D., Cipolotti, L., Husain, M., Vargha-Khadem, F., et al. (2007). The hippocampus is required for short-term topographical memory in humans. Hippocampus, 17, 34-48.

Holdstock, J. S., Mayes, A. R., Roberts, N., Cezayirli, E., Isaac, C. L., O’Reilly, R. C., et al. (2002). Under what conditions is recognition spared relative to recall after selective hippocampal damage in humans? Hippocampus, 12, 341-351.

Intraub, H. (1980). Presentation rate and the representation of briefly glimpsed pictures in memory. Journal of Experimental Psychology: Human Learning and Memory, $6,1-12$.

Jonides, J., Lewis, R. L., Nee, D. E., Lustig, C. A., Berman, M. G., \& Moore, K. S. (2008). The mind and brain of short-term memory. Annual Review of Psychology, 59, 193-224.

Koenigs, M., Barbey, A. K., Postle, B. R., \& Grafman, J. (2009). Superior parietal cortex is critical for the manipulation of information in working memory. The Journal of Neuroscience: The Official Journal of the Society for Neuroscience, 29, 14980-14986.

Konkel, A., Warren, D. E., Duff, M. C., Tranel, D. N., \& Cohen, N. J. (2008). Hippocampal amnesia impairs all manner of relational memory. Frontiers in Human Neuroscience, 2, 15

Lee, A. C., \& Rudebeck, S. R. (2010). Human medial-temporal lobe damage can disrupt the perception of single objects. The Journal of Neuroscience: The Official Journal of the Society for Neuroscience, 30, 6588-6594.

Lee, A. C. H., Buckley, M. J., Pegman, S. J., Spiers, H., Scahill, V. L., Gaffan, D., et al. (2005). Specialization in the medial-temporal lobe for processing of objects and scenes. Hippocampus, 15, 782-797.

Lee, A. C. H., Bussey, T. J., Murray, E. A., Saksida, L. M., Epstein, R. A., Kapur, N., et al. (2005). Perceptual deficits in amnesia: Challenging the medial-temporal lobe "mnemonic" view. Neuropsychologia, 43, 1-11.

Macmillan, N. A., \& Creelman, C. D. (2005). Detection theory: A user's guide (2nd ed.). Mahwah, NJ: Lawrence Erlbaum Associates.

Marr, D., \& Nishihara, H. K. (1978). Representation and recognition of the spatial organization of three-dimensional shapes. Proceedings of the Royal Society of London, Series B, Containing Papers of a Biological Character. Royal Society (Great Britain), 200, 269-294.

Milner, B. (1962). Les trouble de la memoire accompagnant des lesions hippocampiques balaterals. In Psychologie de l'bippocampe (pp. 257-272). Paris: Centre National de la Recherche Scientifique. [English translation: Milner, P. M., \& Glickman S. (Eds.). (1965). Cognitive processes and the brain (pp. 97-111). Princeton: Van Nostrand].

Nichols, E. A., Kao, Y. C., Verfaellie, M., \& Gabrieli, J. D. E. (2006). Working memory and long-term memory for faces: Evidence from fMRI and global amnesia for involvement of the medial-temporal lobes. Hippocampus, 16, 604-616.

Norman, K. A. (2010). How hippocampus and cortex contribute to recognition memory: Revisiting the complementary learning systems model. Hippocampus, 20, 1217-1227.

Norman, K. A., \& O’Reilly, R. C. (2003). Modeling hippocampal and neocortical contributions to recognition memory: A complementary-learning-systems approach. Psychological Review, 110, 611-646.

Olsen, R. K., Nichols, E. A., Chen, J., Hunt, J. F., Glover, G. H., Gabrieli, J. D., et al. (2009). Performance-related sustained and anticipatory activity in human medial-temporal lobe during delayed match-to-sample. The Journal of Neuroscience: The Official Journal of the Society for Neuroscience, 29, 11880-11890.

Olson, I. R., Moore, K. S., Stark, M., \& Chatterjee, A. (2006). Visual working memory is impaired when the medial-temporal lobe is damaged. Journal of Cognitive Neuroscience, 18, 1087-1097.

Olson, I. R., Page, K., Moore, K. S., Chatterjee, A., \& Verfaellie, M. (2006). Working memory for conjunctions relies on the medial-temporal lobe. Journal of Neuroscience, 26, 4596-4601.

Postle, B. R. (2006). Working memory as an emergent property of the mind and brain. Neuroscience, 139, 23-38.

Prisko, L. (1963). Short-term memory in focal cerebral damage. Unpublished PhD, McGill University, Montreal, CA.

Ranganath, C., Cohen, M. X., \& Brozinsky, C. J. (2005). Working memory maintenance contributes to long-term memory formation: Neural and behavioral evidence. Journal of Cognitive Neuroscience, 17, 994-1010.

Ranganath, C., Cohen, M. X., Dam, C., \& D'Esposito, M. (2004). Inferior temporal, prefrontal, and hippocampal contributions to visual working memory maintenance and associative memory retrieval. Journal of Neuroscience, 24, 3917-3925.

Ranganath, C., \& D'Esposito, M. (2001). Medial-temporal lobe activity associated with active maintenance of novel information. Neuron, 31, 865-873.

Rossi, A. F., Pessoa, L., Desimone, R., \& Ungerleider, L. G. (2009). The prefrontal cortex and the executive control of attention. Experimental Brain Research. Experimentelle Hirnforschung. Experimentation Cerebrale, 192, 489-497.

Ryan, J. D., Althoff, R. R., Whitlow, S., \& Cohen, N. J. (2000). Amnesia is a deficit in relational memory. Psychological Science, 11, 454-461. 
Ryan, J. D., \& Cohen, N. J. (2004a). The nature of change detection and online representations of scenes. Journal of Experimental Psychology: Human Perception and Performance, 30, 988-1015.

Ryan, J. D., \& Cohen, N. J. (2004b). Processing and short-term retention of relational information in amnesia. Neuropsychologia, 42, 497-511.

Scoville, W. B., \& Milner, B. (1957). Loss of recent memory after bilateral hippocampal lesions. Journal of Neurology, Neurosurgery \& Psychiatry, 20, 11-21.

Shrager, Y., Levy, D. A., Hopkins, R. O., \& Squire, L. R. (2008) Working memory and the organization of brain systems. Journal of Neuroscience, 28, 4818-4822.

Sidman, M., Stoddard, L. T., \& Mohr, J. P. (1968). Some additional quantitative observations of immediate memory in a patient with bilateral hippocampal lesions. Neuropsychologia, 6, 245-254.
Stern, C. E., Sherman, S. J., Kirchhoff, B. A., \& Hasselmo, M. E. (2001). Medial-temporal and prefrontal contributions to working memory tasks with novel and familiar stimuli. Hippocampus, 11, 337-346.

Wager, T. D., \& Smith, E. E. (2003). Neuroimaging studies of working memory: A meta-analysis. Cognitive, Affective $\varepsilon$ Behavioral Neuroscience, 3, 255-274.

Warren, D. E., Duff, M. C., Tranel, D., \& Cohen, N. J. (2010). Medial-temporal lobe damage impairs representation of simple stimuli. Frontiers in Human Neuroscience, 4, 35.

Wechsler, D. (1997). Wechsler adult intelligence scalethird edition. New York: The Psychological Corporation.

Zanto, T. P., Rubens, M. T., Thangavel, A., \& Gazzaley, A. (2011). Causal role of the prefrontal cortex in top-down modulation of visual processing and working memory. Nature Neuroscience, 14, 656-661. 